2

3

4

5

6

7

8

9

10

11

12

13

14

15

16

17

18

19

20

21

22

23

24

25

26

27

28

29

30

31

32

.

\title{
Licence for Destruction: Tumor-specific Cytokine Targeting
}

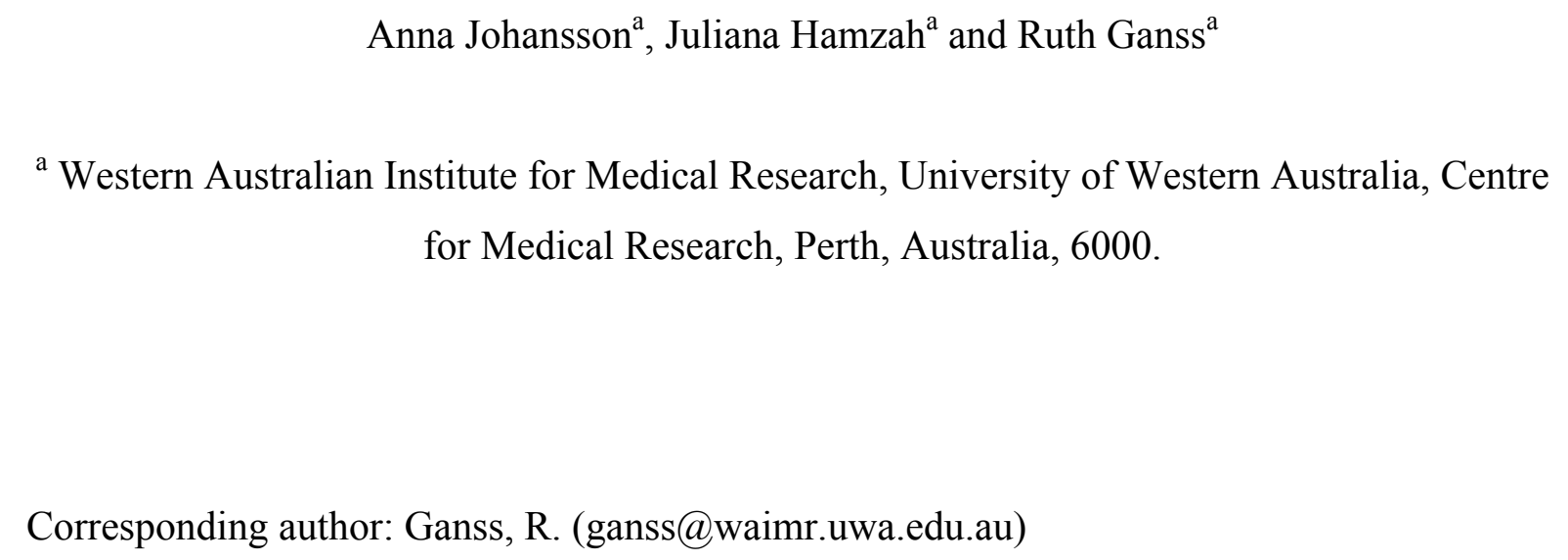

Corresponding author: Ganss, R. (ganss@waimr.uwa.edu.au) 


\section{Abstract}

2 Stroma is an integral part of solid tumors and plays a key role in growth promotion and 3 immune suppression. Most current therapies focus on destroying tumors and/or abnormal 4 vasculature. However, evidence is emerging that anti-cancer efficacy improves with vessel 5 normalization rather than destruction. Specific targeting of cytokines into tumors provides 6 proof-of-concept that tumor stroma is dynamic and can be remodeled to increase drug access 7 and alleviate immune suppression. Changing the inflammatory milieu "opens" tumors for 8 therapy and thus provides the license for destruction. This involves re-programming of 9 paracrine signaling networks between multiple stromal components to break the vicious cycle 10 of angiogenesis and immune suppression. With active immunotherapy rapidly moving into 11 the clinic, local cytokine delivery emerges as an attractive adjuvant.

12 


\section{Glossary:}

2 Cancer immunotherapy: cancer immunotherapy uses the immune system to reject tumors.

3 Only recently, active immunotherapy has become available for cancer patients with the 4 clinical approval of two agents: sipuleucel-T (Provenge, Dendreon) an autologous, dendritic 5 cell vaccine for advanced prostate cancer, and Ipilimumab (Yervoy, Bristol-Myers Squibb) a monoclonal antibody to cytotoxic T-lymphocyte-associated antigen-4 (CTLA-4), for metastatic melanoma.

CD40: CD40 is a costimulatory molecule expressed on antigen presenting cells and in tumor stroma. CD40 agonists activate $\mathrm{T}$ cells and modulate tumor stroma. Anti-tumor effects of agonistic CD40 antibodies are currently assessed in clinical trials.

CpG-ODN: $\mathrm{CpG-ODN}$ is a synthetic reagent which consists of immunostimulatory oligodeoxynucleotides (ODN) with cytosine-guanine-rich (CpG) motifs and a phosphothioate-stabilized backbone. It mimics bacterial DNA and is a potent immune adjuvant. Through its interaction with Toll-like receptor-9 (TLR-9), CpG-ODN activates B cells and plasmacytoid dendritic cells (DC) in humans and a broader spectrum of DCs, B cells, and macrophages in mice.

Hypoxia: tumor hypoxia or low oxygen concentration is a consequence of vascular abnormalities and low oxygen supply in rapidly growing tumors which outgrow their blood supply. Anti-cancer drugs are often unable to penetrate into hypoxic areas.

Interstitial fluid pressure (IFP): IFP is regulated by stromal cells and extracellular matrix.

Solid tumors have a raised IFP due to increased vessel permeability, lymphatic vessel abnormalities and interstitial fibrosis. Increased IFP in tumors reduces perfusion and drug penetration.

Pericytes: specialized mesenchymal cells which line and stabilize endothelial cells of small capillaries. Pericytes are part of the abnormal vascular bed in tumors and often loosely attached to tumor endothelial cells, reduced in numbers or less mature. Pericyte coverage is an important parameter for the assessment of tumor vessel remodeling/normalization. 
1 Phage display libraries: phage display libraries are bacteriophage particles with randomly 2 displayed peptides or antibody fragments of different binding specificities. Each 3 peptide/antibody fragment recognizes different target molecules. Libraries can be injected 4 intravenously to specifically screen for binding activities in a particular tissue or tumor.

5 Phages that bind to target molecules in the tumor of interest are enriched after multiple 6 rounds of biopanning. Subsequently, binding moieties are analysed and can be developed into 7 targeting vehicles.

8

9 Regulator of G protein Signaling-5 (RGS5): RGS5 is a member of the regulator of G protein signaling family which is abundantly expressed in vascular smooth muscle cells and modulates vascular homeostasis by controlling $\mathrm{G}$ protein-coupled receptor signaling. RGS5 is specifically upregulated in tumor pericytes. Removing RGS5 from the tumor microenvironment in murine pancreatic tumors leads to normalization of the tumor vasculature and improved response to immunotherapy.

Tumor-associated macrophages (TAM): innate immune cells which are found in the majority of solid tumors. TAMs represent M2 activated macrophages which promote tumor growth by secreting factors that stimulate breakdown of extracellular matrix and vessel growth, and inhibit anti-cancer immunity. In contrast, M1 macrophages support anti-tumor immunity. 
2 Tumor cells are embedded in stroma which is composed of blood vessels, immune cells, and connective tissue including fibroblasts and extracellular matrix. Stroma is crucially involved in tumor growth, invasion and metastasis [1-2]. The tumor microenvironment also impedes drug delivery and thus reduces the efficacy of conventional anti-tumor therapies such as chemo- and radiation therapy [3-4]; similar mechanisms contribute to a general lack of cytotoxic $\mathrm{T}$ cell function and anti-tumor immunity [5-6].

Tumors create their own microenvironments which are diverse and tumor type- and stagedependent; however, tumors also share common stromal features and signaling themes. In particular, intricate relationships between inflammatory factors, macrophages and blood vessels exist in most solid tumors which modulate growth, therapeutic response and ultimately relapse [7-9]. Disruption of these relationships and remodeling of stroma opens tumors for cytotoxic drugs or immune destruction and thus creates new and exciting opportunities for anti-cancer therapy. As new stromal markers and functional relationships are discovered, potential therapeutic strategies include local delivery of drugs/toxins into the tumor microenvironment via peptides or antibodies. In vivo screening of phage-display libraries of peptides or antibodies have identified unique targets for stroma-specific molecules in situ, resulting in successful development of targeted delivery of diagnostic or bioactive fusion compounds [10-12]. These compounds may have efficacy on their own, but in the right context, can also enhance other anti-cancer drugs and, importantly, anti-tumor immunity. Here, we discuss the latest aspects of "microenvironmental therapy" which exploits the conventional concept of peptide or antibody-mediated targeting in the context of tumor stroma modulation and vascular normalization for improved combination therapies.

\section{Targeting tumor vasculature}

The majority of peptides or antibodies with high stromal affinity identified by in vivo perfusion methods bind to the angiogenic vasculature in solid tumors $[10,11]$ (Box 1). This is not surprising since tumor blood vessels are morphologically and functionally different from normal blood vessels [13]. Moreover, intravenous injection and blood circulation facilitate binding of ligands on the luminal side of endothelial cells. Such peptides which generically home to angiogenic vessels have the RGD (Arg-Gly-Asp) or NGR (Asn-Gly-Arg) motifs which bind to $\alpha_{v} \beta_{3} / \alpha_{v} \beta_{5}$ integrins and the metalloprotease aminopeptidase N (CD13), 
respectively [14-15]. Most prominent vessel-targeting antibody fragments (single-chain variable fragments, $\mathrm{scFv})$ are directed against specific splice variants of fibronectin (L19, F8) and tenascin $\mathrm{C}$ (G11, F16), both part of the extracellular matrix (ECM) surrounding tumor neovasculature [16-19]. Thus, these targeting reagents commonly recognize molecules actively involved in cell-cell/cell-matrix interactions and angiogenic vessel remodeling, and are frequently overexpressed in the tumor vasculature. Importantly, the value of these peptides/antibodies is in their potential to deliver therapeutic payloads into precise tumor stromal compartments.

\section{Therapeutic tumor targeting}

Peptide or antibody fusion compounds when used as carrier molecules provide a unique opportunity to improve anti-cancer therapy whilst reducing harmful side effects. An impressive spectrum of fusion compounds for delivery of toxic agents, radionuclides, procoagulation factors and cytokines have been tested in preclinical tumor models and some clinical trials are underway (Table 1). For instance, application of the NGR motif fused with pro-apoptotic factors such as tumor necrosis factor (TNF)-related apoptosis-inducing ligand (TRAIL) [20] or the D-amino acid peptide ${ }_{\mathrm{D}}[\mathrm{KLAKLAK}]_{2}$ [21] induces tumor endothelial cell apoptosis. Vessel-targeted truncated tissue factor (tTF) causes thrombosis and vessel collapse in animal models and some reduction in tumor perfusion in a clinical case [22]. Chemotherapeutic drugs have also been directly conjugated to targeting moieties or used in various combination therapies [23-24]. Indeed, most targeting efforts to date have been directed to improve cytotoxic drug delivery into tumors and enhance drug penetration into parenchyma to amplify anti-tumor cytotoxicity (Box 2).

Inflammatory factors such as cytokines represent attractive compounds for specific, high dose delivery into tumors. Anti-tumor effects of cytokines have been well documented over the last decades. In particular high-dose TNF $\alpha$ disrupts angiogenic vessels and is currently used in isolated limb perfusion to treat locally advanced melanoma and soft tissue sarcoma [2526]. However, the clinical application of cytokines has been restricted to local treatment due to high toxicity. Precise targeting of tumor vessels is therefore a promising strategy and has been employed for various peptide/antibody-cytokine chimeric compounds which include interleukin (IL) 2, IL12, interferon $\gamma$ (IFN $\gamma$ ) and TNF $\alpha$ (Table 1). Most cytokines are not cytotoxic for cancer cells, but exert direct anti-vascular effects at high doses and also modulate the host's immune system. Thus, tumor rejection in preclinical models requires immune-competency, frequently leads to tumor infiltration by adaptive and innate immune 
cells, and may also induce immunological memory [27-28]. Interestingly, efficacy of targeted cytokine therapy can be enhanced when combined with conventional chemotherapy possibly by promoting intratumoral accumulation of cytotoxic drugs and/or tumor antigen presentation [29-31]. Selected compounds are currently being evaluated in clinical trials with encouraging results especially in combination with chemotherapy (Table 1). Given the clinical application of TNF $\alpha$ in isolated limb perfusion, TNF $\alpha$ is one of the best-studied cytokines which has also been conjugated to ligands such as NGR, RGD and L19, and successfully used as a liganddirected vascular targeting agent (Table 1). Anti-cancer effects of vessel-targeted TNF $\alpha$ were attributed to increased tumor vessel leakage and enhanced drug uptake [29-30, 32]. Whilst these mechanisms are difficult to demonstrate in the clinic [33], low dose NGR-TNF $\alpha$ is currently the only peptide compound with vessel-targeting capability which shows promising effects in early clinical trials when combined with chemotherapy [34-35].

Notably, most selective vascular targeting approaches induce vessel death. Destroying tumor vasculature to restrict blood supply has been validated clinically leading to the approval of several drugs which block vascular endothelial growth factor (VEGF) signaling pathways. When used in combination with chemotherapy, disease stabilization and overall survival benefits are observed for some tumor types. However, whilst destruction of tumor vessels is a logical anti-cancer approach, the optimism of three decades has been tempered by merely transient anti-tumor effects and disappointing long term responses [36]. Conceivably the major value of anti-vascular therapies will only occur by further improving vascular targeting strategies and in combination with synergistic treatment modalities such as chemo- and immunotherapy. Interestingly, it may well be that restoration of tumor vessel function, so called normalization, rather than destruction may be an alternative and more sustained approach to enhance anti-tumor effects of vessel targeting strategies; this requires further investigation to explore its full therapeutic potential.

\section{Tumor targeting of immune modulatory cytokines re-visited}

Earlier studies using NGR-TNF $\alpha$ in conjunction with cytotoxic drugs for anti-cancer therapy were based on the assumption that cytokines such as TNF $\alpha$ damage the tumor vasculature, increase vessel leakiness and thus drug and immune cell penetration [32, 37-38]. However, this is difficult to reconcile with the exceedingly low therapeutically effective doses of NGR$\mathrm{TNF} \alpha$ (nanograms (ng) or picograms in mice [38] and $0.8 \mathrm{microgram}(\mu \mathrm{g}) / \mathrm{m}^{2}$ in humans [3940]). Also, increased vessel leakiness for plasma molecules does not necessarily enhance 
1 drug perfusion deep into solid tumors. Instead, leaky tumor vessels create a hypoxic environment with increased interstitial fluid pressure (IFP) [3]. High IFP in turn acts as a barrier for effective drug delivery and may also prevent infiltration of immune effector cells. This raises the question what other vascular changes may render tumors permissive for drug or immune cell uptake. Cytokines including TNF $\alpha$ activate vessels which play a crucial role for leukocyte trafficking into sites of inflammation and conceivably into tumor vascular beds. Interestingly, $\mathrm{TNF} \alpha$ has also been shown to reduce IFP, and low dose systemic injection facilitates uptake of circulating liposomes indicative of improved blood flow, tumor perfusion and vessel function [41-42]. Indeed, recent studies have re-visited intratumoral cytokine effects and provide new insights how tumor-targeted cytokines may be exploited to activate and/or remodel the tumor vasculature for improved therapy [43-45].

\section{Vascular activation and anti-tumor immunity}

Despite the presence of inflammatory cytokines in solid tumors, transmigration through endothelial barriers and tumor homing of effector $\mathrm{T}$ cells seems to be a rate limiting step for tumor cell lysis [46]. However, a common underlying theme of successful immune therapy is the activation of tumor blood vessels [47]. Indeed, experimental induction of vascular adhesion molecules such as E-/P-selectins, vascular cell adhesion molecule (VCAM) and intercellular adhesion molecule (ICAM) in tumor vascular beds enhances $\mathrm{T}$ cell transmigration and tumor rejection [48-53]. For instance, in ovarian cancer, expression of endothelin B receptor (ETBR) on tumor vessels is inversely correlated with tumor infiltrating lymphocytes and patient survival. Inhibition of ETBR increases ICAM-1 expression on endothelia and concomitantly $\mathrm{T}$ cell influx [49]. Similarly, inert tumor vessels in several tumor models are rendered susceptible for $\mathrm{T}$ cell penetration and immune-mediated tumor killing by IL-6-dependent induction of inflammatory adhesion molecules such as E/P-selectin and ICAM-1 [53]. Thus, anergic tumor blood vessels can be activated to permit immune cell invasion and tumor cell killing. Intriguingly, low dose TNF $\alpha$ when targeted to tumor vessels in a mouse model of pancreatic endocrine tumors activates the vasculature resulting in high expression of adhesion molecules. This enables effector cell trafficking into tumor parenchyma, but more importantly, shows strong therapeutic effects when combined with anti-tumor vaccination or adoptive $\mathrm{T}$ cell transfers [45]. Thus, vascular activation induced by changing the intratumoral cytokine profile can act as a strong adjuvant to immunotherapy (Table 2). Moreover, synergistic efficacy of a triple therapy combining low dose NGR-TNF $\alpha$ 
with anti-tumor vaccination and chemotherapy is observed in a mouse model of subcutaneously growing melanoma [54]. These studies demonstrate that a combination of cytokine targeting with immunotherapy is a highly effective approach; in combination with chemotherapy, induction of immunogenic cancer cell death may further enhance activation of anti-tumor immunity [55]. This warrants further investigation in particular in light of increasing clinical applications of anti-cancer immune therapy [56].

7

\section{8}

\section{Vascular normalization and anti-tumor immunity}

Recently, a new concept has emerged where vessel stabilization and reduced vascular permeability, a phenomenon described as vessel normalization [57], improves anti-cancer therapies; remarkably, this includes chemo- and radiation therapies, delivery of nanoparticles and immunotherapy [50, 58-63]. Proof-of-principle that vessel remodeling per se, even in the absence of inflamed vascular endothelium, dramatically improves the outcome of immunotherapy comes from genetic deletion of the regulator of G protein signaling (RGS) 5 . This gene is highly upregulated in angiogenic vessels; loss of RGS5 causes pericyte maturation, vascular normalization and importantly, activated $\mathrm{T}$ effector cell influx in quantities sufficient for tumor rejection in a mouse model of pancreatic endocrine cancer [50]. Subsequently, it has been demonstrated that blockade of VEGF/VEGFR pathways for instance in B16 melanoma and orthotopic breast cancer models temporarily normalizes vessels and improves active immunotherapy, in particular with low dose angiostatic treatment $[60,63]$. It is conceivable that improved tumor blood flow concomitant with reduced hypoxia and IFP enhances delivery of therapeutics including tumor-specific T cells.

In preclinical models, evidence for enhanced therapeutic efficacy with vessel modulation and normalization rather than destruction is compelling. Ongoing clinical studies show promising synergy between angiostatic treatment and chemotherapy. Whether this is the result of vessel destruction and increased leakiness or vessel remodeling and improved perfusion is still unclear. So far, only a limited number of clinical studies have been conducted which assess tumor perfusion/vessel permeability in the context of classical anti-angiogenesis therapy [64]. Currently, NGR-TNF $\alpha$ is the only targeting compound where imaging of vascular changes was incorporated in a clinical trial. In a patient cohort undergoing a phase I dose escalation study, dynamic contrast enhanced (DCE)-MRI was used to analyse early effects $(2 \mathrm{~h})$ of NGR-TNF $\alpha$ on vessel permeability [33]. Overall, DCE-MRI failed to predict the optimal treatment dose. However, interesting observations include heterogeneity in vascular response 
depending on tumor size, e.g., small nodules are more responsive. Also observed are reduced leakiness of vessels in liver metastases after treatment, which may be indicative of vascular normalization, and off-target effects on normal liver tissue despite a tumor-specific vascular targeting approach. Since animal studies document the dynamic nature of drug-induced vessel remodeling (Figure 1) imaging of acute, disease stabilizing and relapse phases for different human tumors is warranted in particular in anticipation of immune combination therapies.

\section{Vessel modulation is context-dependent}

Changes in tumor vessels, be it death, activation or normalization, are not mutually exclusive. Instead, modulation of the tumor vasculature is a dynamic process which can induce several phenotypes simultaneously or consecutively (Figure 1). Moreover, the actual vascular effects of peptide targeted inflammatory mediators vary significantly depending on compound, dose, and treatment duration. This is exemplified in a series of studies which used a prototypic mouse model of pancreatic endocrine cancer and the RGR peptide to correlate vascular effects with anti-tumor immunity (Figure 1). In this model, even fully activated anti-tumor effector cells fail to penetrate highly angiogenic tumors as is often observed in human cancers. Interestingly, vascular targeting of $\mu \mathrm{g}$ quantities of IFN $\gamma$ destroys the tumor vasculature resulting in some therapeutic efficacy reminiscent of anti-angiogenic therapy; intratumoral IFN $\gamma$ fails, however, to improve $\mathrm{T}$ cell extravasation and anti-tumor immune responses [45]. Even though IFN $\gamma$ has potentially strong immune-enhancing properties, it predominantly affects angiogenesis in the tumor microenvironment. This is consistent with other models where IFN $\gamma$ specifically destroys stroma during immune-mediated tumor rejection [65]. Thus, cytokines with strong anti-vascular effects do not necessarily enhance leukocyte influx and tumor immunity. Similarly, no synergy is observed between vascular disruption agents which cause hemorrhagic tumor necrosis and active anti-cancer immune therapy [66]. In contrast, agonistic CD40 antibodies when conjugated with RGR peptide specifically activate CD40-positive tumor vessels to express VCAM, ICAM and E-selectin. Activation of endothelia alone has no anti-tumor efficacy. However, when combined with adoptive transfers of anti-tumor $\mathrm{T}$ cells, these effector cells efficiently enter otherwise inaccessible tumors. Survival was further enhanced by using an IL2-RGR fusion protein which increases effector cell activity once they reach the tumor site [43]. Effective vessel activation is also achieved by vascular targeting of RGR-coated liposomes containing toll- 
1 like receptor (TLR) 9 ligands (e.g., oligodeoxynucleotides with CpG motifs, CpG-ODNs).

2 Remarkably, CpG-ODN not only inflames vessel walls but also primes anti-tumor cytotoxicity, most likely due to uptake by intratumoral macrophages and efficient antigen presentation. Again, spontaneous anti-tumor immunity is further enhanced with adoptively transferred anti-tumor T cells [44]. As discussed above, low dose RGR-TNF $\alpha$ therapy (ng and $\mu \mathrm{g}$ quantities) induces strong expression of inflammatory adhesion molecules on tumor vessels. Simultaneously, vessels are stabilized with reduced permeability and increased perfusion. This stage also permits influx of adoptively transferred, pre-activated effector cells. However, continuous, low dose RGR-TNF $\alpha$ kills vessels and stroma and ultimately limits effector cell penetration [45]. Stromal destruction as a consequence of prolonged treatment is observed in most preclinical models and demonstrates the unsustained nature of current treatment modalities [43-44, 57]. Thus, the challenge ahead is to develop targeting strategies which modulate rather than destroy stroma for improved anti-tumor immunity.

\section{Immune-amplification in the tumor environment}

Vessel targeting strategies naturally focus on direct changes in the tumor vascular bed. However, recent evidence demonstrates an intricate network of paracrine signals between vessels and other stromal cells, most notably macrophages, which indirectly shapes the vascular phenotype, and the tumor immune milieu. Tumor-associated macrophages (TAMs) display a high degree of plasticity and have been implicated in vascular remodeling. TAMs normally exhibit an M2 phenotype and, without intervention, foster angiogenesis, tumor growth and immune evasion [9]. However, TAMs can be re-educated to reverse the tumor promoting program [45, 67-69]. For instance, genetic deletion of VEGF specifically in myeloid cells results in vascular normalization in a transgenic mouse model of breast cancer which in turn increases susceptibility to chemotherapy [67]. Experimental tumors with forced expression of histidine-rich glycoprotein harbor M1-type macrophages which produce less placental growth factor (PlGF) and display a normalized vasculature; re-programming of TAMs improves chemotherapy response and also anti-tumor immunity [69]. Moreover, IFN $\alpha^{\text {high }}$ myeloid cells which specifically home to tumor vessels in an orthotopic glioma model stimulate TAMs to secrete proinflammatory cytokines such as IL-1 and TNF $\alpha$ concomitant with vascular normalization [68].

Intriguingly, TAMs also play a crucial role in therapeutic efficacy of low dose TNF $\alpha$ targeting. Besides vessel remodeling, intratumoral RGR-TNF $\alpha$ elicits profound stromal 
activation which also includes TAMs. TAMs change their expression profile after TNF $\alpha$ treatment and are polarized to secrete M1-like inflammatory factors, e.g., monocyte chemotattractant protein (MCP)-1, inducible nitric oxide synthase (iNOS), and angiopoietin (Ang)2. This in turn has dual effects on the tumor environment: first, M1-like TAMs act as a strong adjuvant to $\mathrm{CD}^{+} \mathrm{T}$ cell effector function, and second, Ang2 in conjunction with TNF $\alpha$ upregulates the expression of endothelial adhesion molecules which in turn facilitated leukocyte transmigration [45] (Figure 2). This immune amplification cascade which triggers profound changes in the tumor micromilieu explains some of the remarkable effects of low dose intratumoral TNF $\alpha$. It also provides proof-of-concept that although vascular targeting has obvious limitations, such as non-specific uptake, heterogeneous binding to tumor vessels and insufficient payload delivery, cytokine targeting is a viable and promising approach for further exploration.

\section{Concluding remarks and future perspective}

Over the past decade tumor targeting has been explored and refined concomitant with increasing knowledge about tumors and their microenvironments. Whilst the idea of fighting tumors from the inside is compelling, it is transpiring that therapeutic use of tumor targeting will depend on strategies to maximize intratumoral effects. This includes optimized bioengineering for improved ligand-receptor interactions, use of targeting moieties which allow deeper and more homogeneous access into the tumor parenchyma, and simultaneous or sequential targeting of multiple stromal components. Discovery of new ligands which bind to non-vascular stromal targets such as cancer-associated macrophages and fibroblasts [70-71] will facilitate this process. Multi-targeting strategies could then be explored to eliminate or remodel stromal cells in primary tumors and also metastatic lesions [72].

Ultimately, however, stromal targeting requires further refinement to enhance other anticancer modalities. Vascular destruction without complete eradication of cancer cells will at best delay tumor growth but may also induce resistance, relapse and increased invasiveness as witnessed with anti-angiogenesis drugs. New approaches which exploit the dynamic nature of tumor stroma to re-program rather than destroy vessels may provide longer lasting antitumor effects, in particular, if stromal remodeling is combined with strategies to eliminate cancer cells such as cytotoxic, radiation, molecular targeted and immune therapies.

Exciting new developments demonstrate that immune signaling cascades can be activated to amplify anti-tumor activity by re-education of tumor-resident cells and recruitment of 
1 immune cells into tumors which promote rejection [44]. In this context it is remarkable that 2 minute amounts of selected inflammatory factors when delivered into tumors can have 3 profound effects on both tumor perfusion and anti-tumor immunity [45]. This is consistent

4 with an active immune-suppressive role of stroma and the observation that soluble factors in

5 untreated tumors simultaneously promote angiogenesis and immunosuppression. Overall, this

6 encourages further development of combination therapies which first create an angiostatic

7 and immunostimulatory environment followed by cell lysis for complete tumor destruction.

8

\section{Acknowledgements}

10 This work was supported by Cancer Council Western Australia, Medical Research 11 Foundation Royal Perth Hospital, and the National Health and Medical Research Council. 


\section{Box 1. Tumor peptide-targeting up to date}

2 Binding activities of peptide-ligands can be based on overexpression and also on cancerspecific cellular localization of their receptors which differs from normal cells. For example, the F3 peptide, a fragment of the human high mobility group protein 2, binds to nucleolin which can be aberrantly exposed on the surface of endothelial and tumor cells during carcinogenesis [73]. Tumor endothelial cells are surrounded by pericytes and a basement membrane, both of which are commonly altered in solid tumors. Collagen IV for instance is modified by matrix metalloproteinases (MMPs) during angiogenic vessel remodeling and a peptide sequence (TLTYTWS) has been identified that specifically binds to collagen IV modified by MMP-2 [74]. Peptide ligands such as CPRECES (receptor: aminopeptidase A (CD249)), CRGRRST (RGR peptide, putative receptor: platelet-derived growth factor receptor (PDGFR) $\beta)$, CSRNLIDC (pBP peptide, receptor: PDGFR $\beta$ ) or indeed NGR (receptor CD13) with vascular targeting properties may also bind to tumor pericytes [54, 7578]. As a consequence of leaky tumor blood vessels, blood clotting complexes are located in tumor vessels and surrounding stroma which provide specific docking signals for the pentapeptide CREKA [79]. Besides blood vessels, lymphatic vessels are also an integral part of solid tumors and intimately involved in metastatic spread. Peptides such as LyP-1 (CGNKRTRGC) which have been identified as ligands for the molecule p32, when expressed on the cell surface, bind to tumor-associated lymphatics, some p32-positive tumor cells and tumor-associated macrophages [70].

\section{Box 2. Vascular homing peptides: optimizing cytotoxic anti-tumor effects}

One strategy to improve vascular targeting and anti-tumor effects is to use nanoparticles decorated with multiple vascular homing peptides and loaded with cytotoxic drugs for release at the tumor site [11]. Multivalent targeting of cytotoxic nano-carriers can overcome some pharmacological limitations of directly conjugated peptide ligands. However, access into tumors beyond the vasculature remains challenging. Recently, Ruoslahti and colleagues described a series of peptides which specifically bind to tumor stroma and also penetrate into tumor tissue [94-96]. Most remarkable is the capacity of prototypic iRGD peptide (CRGDK/RGPD/EC) to deliver payloads such as doxorubicin into tumors simply by coadministration, thus abolishing the need to produce fusion compounds. This approach results in a 14-fold increase in doxorubicin containing liposomes in tumors as compared to injection of doxorubicin liposomes without iRGD and significantly enhances anti-tumor effects [94]. 
1 Another recent development elegantly harnesses biological effector cascades to combine 2 traditional chemotherapy with vascular destruction. For instance, rod-shaped gold 3 nanoparticles (nanorods) which passively home into tumors are used to induce coagulation 4 under near-infrared light irradiation. This in turn significantly enhances accumulation of 5 doxorubicin-loaded liposomes conjugated with the peptide substrate for the coagulation 6 factor FXIII in human breast cancer xenotransplants [97]. Specific targeting of coagulated 7 tumor vessels for delivery of cytotoxic drugs may overcome some limitations arising with 8 anti-angiogenic therapy such as decreased tumor perfusion and limited drug access. 
3 Figure 1: Dynamic vessel remodeling is context dependent. Solid tumors create a proinflammatory, pro-angiogenic environment which promotes angiogenesis, tumor growth and immune suppression. However, the tumor microenvironment can be modulated by targeted delivery of inflammatory factors with different therapeutic implications. In a mouse model of pancreatic endocrine cancer, targeting of IFN $\gamma$ specifically to the angiogenic vasculature using the RGR peptide results in vessel death and reduced tumor growth without immune involvement [44]. Tumor-targeted agonistic antibodies against CD40 (RGR- $\alpha$ CD40) or inflammatory agents such as RGR-CpG-ODN predominantly activate tumor vessels which in turn promotes effector cell infiltration and immune-mediated tumor regression [42-43]. RGR-TNF $\alpha$ activates and normalizes tumor vessels, and also supports anti-tumor immunity [44]. Vessel activation and normalization are not mutually exclusive. However, most long term treatment with vessel-activating agents leads to stromal destruction which ultimately limits therapeutic efficacy [42-44]. Abbreviations: CD31, vascular marker (also known as platelet endothelial cell adhesion molecule (PECAM-1); CpG-ODN, oligodeoxynucleotides with CpG motifs; IFN $\gamma$, interferon $\gamma$; RGR, peptide sequence (CRGRRST) which confers specific binding to angiogenic tumor vessels; TNF $\alpha$, tumor necrosis factor $\alpha$; VCAM, vascular cell adhesion molecule.

Figure 2: Intratumoral immune amplification. Local, low dose TNF $\alpha$ acts on multiple stromal cells, including blood vessels and macrophages, to improve tumor perfusion, leukocyte extravasation and anti-tumor immunity. TNF $\alpha$-stimulated macrophages, which cluster around the vasculature and play an important role in amplifying vessel activation by secreting Ang2. Ang2, in conjunction with TNFa, upregulates the expression of endothelial adhesion molecules such as VCAM. TNFa also skews tumor-resident macrophages to produce M1-like factors which support T cell activity (MCP1, IL6, iNOS). Thus, immune amplification triggered by tumor- targeted TNF $\alpha$ enhances spontaneous anti-tumor immunity and active immunotherapy [45]. Abbreviations: Ang2, angiopoietin 2; IL6, interleukin 6; iNOS, inducible nitric oxide synthase; MCP1, monocyte chemoattractant protein 1, TNF $\alpha$, tumor necrosis factor $\alpha$, VCAM, vascular cell adhesion molecule. 


\section{References}

21 Egeblad, M., et al. (2010) Tumors as organs: complex tissues that interface with the 3 entire organism. Dev Cell 18, 884-901

42 Engels, B., et al. (2012) Targeting stroma to treat cancers. Semin Cancer Biol 22, 41-49

53 Heldin, C.H., et al. (2004) High interstitial fluid pressure - an obstacle in cancer therapy.

$6 \quad$ Nat Rev Cancer 4, 806-813

74 Correia, A.L. and Bissell, M.J. (2012) The tumor microenvironment is a dominant force 8 in multidrug resistance. Drug Resist Updat 15, 39-49

95 Kraman, M., et al. (2010) Suppression of antitumor immunity by stromal cells 10 expressing fibroblast activation protein-alpha. Science 330, 827-830

116 Johansson, A. and Ganss, R. (2012) Remodeling of tumor stroma and response to 12 therapy. Cancers 4, 340-353

137 Balkwill, F.R. and Mantovani, A. (2012) Cancer-related inflammation: common themes 14 and therapeutic opportunities. Semin Cancer Biol 22, 33-40

158 Nakasone, E.S., et al. (2012) Imaging tumor-stroma interactions during chemotherapy 16 reveals contributions of the microenvironment to resistance. Cancer Cell 21, 488-503

179 Ruffell, B., et al. (2012) Differential macrophage programming in the tumor 18 microenvironment. Trends Immunol 33, 119-126

1910 Schliemann, C. and Neri, D. (2007) Antibody-based targeting of the tumor vasculature. Biochim Biophys Acta 1776, 175-192

11 Ruoslahti, E. (2012) Peptides as targeting elements and tissue penetration devices for nanoparticles. Adv Mater 24, 3747-3756

12 Shetty, D., et al. (2012) Stroma targeting nuclear imaging and radiopharmaceuticals. Int J Mol Imaging 2012, 817682

13 Potente, M., et al. (2011) Basic and therapeutic aspects of angiogenesis. Cell 146, 873887

14 Pasqualini, R., et al. (1997) alpha v Integrins as receptors for tumor targeting by circulating ligands. Nature Biotechnology 15, 542-546

15 Pasqualini, R., et al. (2000) Aminopeptidase $\mathrm{N}$ is a receptor for tumor-homing peptides and a target for inhibiting angiogenesis. Cancer Research 60, 722-727

16 Carnemolla, B., et al. (1996) Phage antibodies with pan-species recognition of the oncofoetal angiogenesis marker fibronectin ED-B domain. Int J Cancer 68, 397-405 
17 Villa, A., et al. (2008) A high-affinity human monoclonal antibody specific to the alternatively spliced EDA domain of fibronectin efficiently targets tumor neovasculature in vivo. Int J Cancer 122, 2405-2413

18 Silacci, M., et al. (2006) Human monoclonal antibodies to domain C of tenascin-C selectively target solid tumors in vivo. Protein Eng Des Sel 19, 471-478

19 Brack, S.S., et al. (2006) Tumor-targeting properties of novel antibodies specific to the large isoform of tenascin-C. Clin Cancer Res 12, 3200-3208

20 Cao, L., et al. (2008) Enhancement of antitumor properties of TRAIL by targeted delivery to the tumor neovasculature. Mol Cancer Ther 7, 851-861

21 Ellerby, H.M., et al. (1999) Anti-cancer activity of targeted pro-apoptotic peptides. Nat Med 5, 1032-1038

22 Bieker, R., et al. (2009) Infarction of tumor vessels by NGR-peptide-directed targeting of tissue factor: experimental results and first-in-man experience. Blood 113, 5019-5027

23 Arap, W., et al. (1998) Cancer treatment by targeted drug delivery to tumor vasculature in a mouse model. Science 279, 377-380

24 Dings, R.P., et al. (2010) Inhibiting tumor growth by targeting tumor vasculature with galectin-1 antagonist anginex conjugated to the cytotoxic acylfulvene, 6hydroxylpropylacylfulvene. Bioconjug Chem 21, 20-27

25 Lejeune, F.J., et al. (2006) Efficiency of recombinant human TNF in human cancer therapy. Cancer Immun 6, 6

26 Seinen, J.M. and Hoekstra, H.J. (2013) Isolated limb perfusion of soft tissue sarcomas: A comprehensive review of literature. Cancer Treat Rev 39, 569-577

27 Curnis, F., et al. (2000) Enhancement of tumor necrosis factor alpha antitumor immunotherapeutic properties by targeted delivery to aminopeptidase $\mathrm{N}$ (CD13). Nat Biotechnol 18, 1185-1190

28 Balza, E., et al. (2010) Therapy-induced antitumor vaccination in neuroblastomas by the combined targeting of IL-2 and TNFalpha. Int J Cancer 127, 101-110

29 Ronca, R., et al. (2009) Delivering cytokines at tumor site: The immunocytokineconjugated anti-EDB-fibronectin antibody case. Immunobiology 214, 800-810

30 Corti, A., et al. (2012) Targeted drug delivery and penetration into solid tumors. Med Res Rev 32, 1078-1091

31 Ma, Y., et al. (2013) Anticancer chemotherapy-induced intratumoral recruitment and differentiation of antigen-presenting cells. Immunity 38, 729-741 
132 van Laarhoven, H.W., et al. (2006) Effects of the tumor vasculature targeting agent NGR-TNF on the tumor microenvironment in murine lymphomas. Invest New Drugs 24, $27-36$

33 Desar, I.M., et al. (2011) Factors affecting the unexpected failure of DCE-MRI to determine the optimal biological dose of the vascular targeting agent NGR-hTNF in solid cancer patients. Eur J Radiol 80, 655-661

34 Gregorc, V., et al. (2009) Phase Ib study of NGR-hTNF, a selective vascular targeting agent, administered at low doses in combination with doxorubicin to patients with

35 Lorusso, D., et al. (2012) Phase II study of NGR-hTNF in combination with doxorubicin in relapsed ovarian cancer patients. Br J Cancer 107, 37-42

36 Ebos, J.M. and Kerbel, R.S. (2011) Antiangiogenic therapy: impact on invasion, disease progression, and metastasis. Nat Rev Clin Oncol 8, 210-221

37 Curnis, F., et al. (2002) Improving chemotherapeutic drug penetration in tumors by vascular targeting and barrier alteration. J Clin Invest 110, 475-482

38 Sacchi, A., et al. (2006) Synergistic antitumor activity of cisplatin, paclitaxel, and gemcitabine with tumor vasculature-targeted tumor necrosis factor-alpha. Clin Cancer Res $12,175-182$

39 Gregorc, V., et al. (2010) Phase II study of asparagine-glycine-arginine-human tumor necrosis factor alpha, a selective vascular targeting agent, in previously treated patients with malignant pleural mesothelioma. J Clin Oncol 28, 2604-2611

40 Santoro, A., et al. (2010) Activity and safety of NGR-hTNF, a selective vasculartargeting agent, in previously treated patients with advanced hepatocellular carcinoma. Br J Cancer 103, 837-844

41 Kristensen, C.A., et al. (1996) Reduction of interstitial fluid pressure after TNF-alpha treatment of three human melanoma xenografts. Br J Cancer 74, 533-536

42 Seynhaeve, A.L., et al. (2007) Tumor necrosis factor alpha mediates homogeneous distribution of liposomes in murine melanoma that contributes to a better tumor response. Cancer Res 67, 9455-9462

43 Hamzah, J., et al. (2008) Vascular targeting of anti-CD40 antibodies and IL-2 into autochthonous tumors enhances immunotherapy in mice. J Clin Invest 118, 1691-1699

44 Hamzah, J., et al. (2009) Targeted liposomal delivery of TLR9 ligands activates spontaneous antitumor immunity in an autochthonous cancer model. J Immunol 183, 1091-1098 
45 Johansson, A., et al. (2012) Tumor-targeted TNFalpha stabilizes tumor vessels and enhances active immunotherapy. Proc Natl Acad Sci U S A 109, 7841-7846

46 Ryschich, E., et al. (2002) Transformation of the microvascular system during multistage tumorigenesis. Int J Cancer 97, 719-725

47 Ganss, R., et al. (2004) Mini-review: overcoming tumor-intrinsic resistance to immune effector function. Eur J Immunol 34, 2635-2641

48 Garbi, N., et al. (2004) CpG motifs as proinflammatory factors render autochthonous tumors permissive for infiltration and destruction. J Immunol 172, 5861-5869

49 Buckanovich, R.J., et al. (2008) Endothelin B receptor mediates the endothelial barrier to T cell homing to tumors and disables immune therapy. Nat Med 14, 28-36

50 Hamzah, J., et al. (2008) Vascular normalization in Rgs5-deficient tumours promotes immune destruction. Nature 453, 410-414

51 Huang, X., et al. (2010) Lymphoma endothelium preferentially expresses Tim-3 and facilitates the progression of lymphoma by mediating immune evasion. J Exp Med 207, 505-520

52 Dings, R.P., et al. (2011) Enhancement of T-cell-mediated antitumor response: angiostatic adjuvant to immunotherapy against cancer. Clin Cancer Res 17, 3134-3145

53 Fisher, D.T., et al. (2011) IL-6 trans-signaling licenses mouse and human tumor microvascular gateways for trafficking of cytotoxic T cells. J Clin Invest 121, 3846-3859

54 Calcinotto, A., et al. (2012) Targeting TNF-alpha to neoangiogenic vessels enhances lymphocyte infiltration in tumors and increases the therapeutic potential of immunotherapy. J Immunol 188, 2687-2694

55 Kroemer, G., et al. (2013) Immunogenic cell death in cancer therapy. Annu Rev Immunol $31,51-72$

56 Sharma, P., et al. (2011) Novel cancer immunotherapy agents with survival benefit: recent successes and next steps. Nat Rev Cancer 11, 805-812

57 Carmeliet, P. and Jain, R.K. (2011) Principles and mechanisms of vessel normalization for cancer and other angiogenic diseases. Nat Rev Drug Discov 10, 417-427

58 Winkler, F., et al. (2004) Kinetics of vascular normalization by VEGFR2 blockade governs brain tumor response to radiation: role of oxygenation, angiopoietin-1, and matrix metalloproteinases. Cancer Cell 6, 553-563

59 Mazzone, M., et al. (2009) Heterozygous deficiency of PHD2 restores tumor oxygenation and inhibits metastasis via endothelial normalization. Cell 136, 839-851 
160 Shrimali, R.K., et al. (2010) Antiangiogenic agents can increase lymphocyte infiltration into tumor and enhance the effectiveness of adoptive immunotherapy of cancer. Cancer Res 70, 6171-6180

61 Wong, C.S., et al. (2012) Vascular normalization by loss of Siah2 results in increased chemotherapeutic efficacy. Cancer Res 72, 1694-1704

62 Chauhan, V.P., et al. (2012) Normalization of tumour blood vessels improves the delivery of nanomedicines in a size-dependent manner. Nat Nanotechnol 7, 383-388

63 Huang, Y., et al. (2012) Vascular normalizing doses of antiangiogenic treatment reprogram the immunosuppressive tumor microenvironment and enhance immunotherapy. Proc Natl Acad Sci U S A 109, 17561-17566

64 Goel, S., et al. (2011) Normalization of the vasculature for treatment of cancer and other diseases. Physiol Rev 91, 1071-1121

65 Qin, Z., et al. (2003) A critical requirement of interferon gamma-mediated angiostasis for tumor rejection by CD8+ T cells. Cancer Res 63, 4095-4100

66 Matthews, K.E., et al. (2006) 5,6-Dimethylxanthenone-4-acetic acid treatment of a nonimmunogenic tumour does not synergize with active or passive CD8+ T-cell immunotherapy. Immunol Cell Biol 84, 383-389

67 Stockmann, C., et al. (2008) Deletion of vascular endothelial growth factor in myeloid cells accelerates tumorigenesis. Nature 456, 814-818

68 De Palma, M., et al. (2008) Tumor-targeted interferon-alpha delivery by Tie2-expressing monocytes inhibits tumor growth and metastasis. Cancer Cell 14, 299-311

69 Rolny, C., et al. (2011) HRG inhibits tumor growth and metastasis by inducing macrophage polarization and vessel normalization through downregulation of PlGF. Cancer Cell 19, 31-44

70 Fogal, V., et al. (2008) Mitochondrial/cell-surface protein $\mathrm{p} 32 / \mathrm{gC} 1 \mathrm{qR}$ as a molecular target in tumor cells and tumor stroma. Cancer Res 68, 7210-7218

71 Brennen, W.N., et al. (2012) Targeting carcinoma-associated fibroblasts within the tumor stroma with a fibroblast activation protein-activated prodrug. J Natl Cancer Inst $104,1320-1334$

72 Yan, Z., et al. (2012) LyP-1-conjugated PEGylated liposomes: a carrier system for targeted therapy of lymphatic metastatic tumor. J Control Release 157, 118-125

73 Christian, S., et al. (2003) Nucleolin expressed at the cell surface is a marker of endothelial cells in angiogenic blood vessels. J Cell Biol 163, 871-878 
74 Mueller, J., et al. (2009) Targeting of tumor blood vessels: a phage-displayed tumorhoming peptide specifically binds to matrix metalloproteinase-2-processed collagen IV and blocks angiogenesis in vivo. Mol Cancer Res 7, 1078-1085

75 Marchio, S., et al. (2004) Aminopeptidase A is a functional target in angiogenic blood vessels. Cancer Cell 5, 151-162

76 Joyce, J.A., et al. (2004) Cathepsin cysteine proteases are effectors of invasive growth and angiogenesis during multistage tumorigenesis. Cancer Cell 5, 443-453

77 Loi, M., et al. (2010) Combined targeting of perivascular and endothelial tumor cells enhances anti-tumor efficacy of liposomal chemotherapy in neuroblastoma. J Control Release 145, 66-73

78 Prakash, J., et al. (2010) A novel approach to deliver anticancer drugs to key cell types in tumors using a PDGF receptor-binding cyclic peptide containing carrier. J Control Release 145, 91-101

79 Simberg, D., et al. (2007) Biomimetic amplification of nanoparticle homing to tumors. Proc Natl Acad Sci U S A 104, 932-936

80 Curnis, F., et al. (2004) Coupling tumor necrosis factor-alpha with alphaV integrin ligands improves its antineoplastic activity. Cancer Res 64, 565-571

81 Dickerson, E.B., et al. (2004) Enhancement of the antiangiogenic activity of interleukin12 by peptide targeted delivery of the cytokine to alphavbeta3 integrin. Mol Cancer Res 2, 663-673

82 Yoshimoto, M., et al. (2008) alpha(v)beta(3) Integrin-targeting radionuclide therapy and imaging with monomeric RGD peptide. Int J Cancer 123, 709-715

83 Bertilaccio, M.T., et al. (2008) Vasculature-targeted tumor necrosis factor-alpha increases the therapeutic index of doxorubicin against prostate cancer. Prostate 68, 1105 1115

84 Schliemann, C., et al. (2009) Complete eradication of human B-cell lymphoma xenografts using rituximab in combination with the immunocytokine L19-IL2. Blood $113,2275-2283$

85 Moschetta, M., et al. (2012) Paclitaxel enhances therapeutic efficacy of the F8-IL2 immunocytokine to EDA-fibronectin-positive metastatic human melanoma xenografts. Cancer Res 72, 1814-1824

86 Balza, E., et al. (2006) Targeted delivery of tumor necrosis factor-alpha to tumor vessels induces a therapeutic $\mathrm{T}$ cell-mediated immune response that protects the host against syngeneic tumors of different histologic origin. Clin Cancer Res 12, 2575-2582 
187 Mortara, L., et al. (2007) Therapy-induced antitumor vaccination by targeting tumor necrosis factor alpha to tumor vessels in combination with melphalan. Eur J Immunol 37, $3381-3392$

88 Pasche, N., et al. (2012) The antibody-based delivery of interleukin-12 to the tumor neovasculature eradicates murine models of cancer in combination with paclitaxel. Clin Cancer Res 18, 4092-4103

89 Gafner, V., et al. (2006) An engineered antibody-interleukin-12 fusion protein with enhanced tumor vascular targeting properties. Int J Cancer 119, 2205-2212

90 Santoro, A., et al. (2010) Phase II study of NGR-hTNF, a selective vascular targeting agent, in patients with metastatic colorectal cancer after failure of standard therapy. Eur $J$ Cancer 46, 2746-2752

91 Mammoliti, S., et al. (2011) Two doses of NGR-hTNF in combination with capecitabine plus oxaliplatin in colorectal cancer patients failing standard therapies. Ann Oncol 22, 973-978

92 Spitaleri, G., et al. (2013) Phase I/II study of the tumour-targeting human monoclonal antibody-cytokine fusion protein L19-TNF in patients with advanced solid tumours. $J$ Cancer Res Clin Oncol 139, 447-455

93 Eigentler, T.K., et al. (2011) A dose-escalation and signal-generating study of the immunocytokine L19-IL2 in combination with dacarbazine for the therapy of patients with metastatic melanoma. Clin Cancer Res 17, 7732-7742

94 Sugahara, K.N., et al. (2010) Coadministration of a tumor-penetrating peptide enhances the efficacy of cancer drugs. Science 328, 1031-1035

95 Agemy, L., et al. (2011) Targeted nanoparticle enhanced proapoptotic peptide as potential therapy for glioblastoma. Proc Natl Acad Sci U S A 108, 17450-17455

96 Roth, L., et al. (2012) Transtumoral targeting enabled by a novel neuropilin-binding peptide. Oncogene $31,3754-3763$

97 von Maltzahn, G., et al. (2011) Nanoparticles that communicate in vivo to amplify tumour targeting. Nat Mater 10, 545-552

98 Schwager, K., et al. (2013) The immunocytokine L19-IL2 eradicates cancer when used in combination with CTLA-4 blockade or with L19-TNF. J Invest Dermatol 133, 751758 


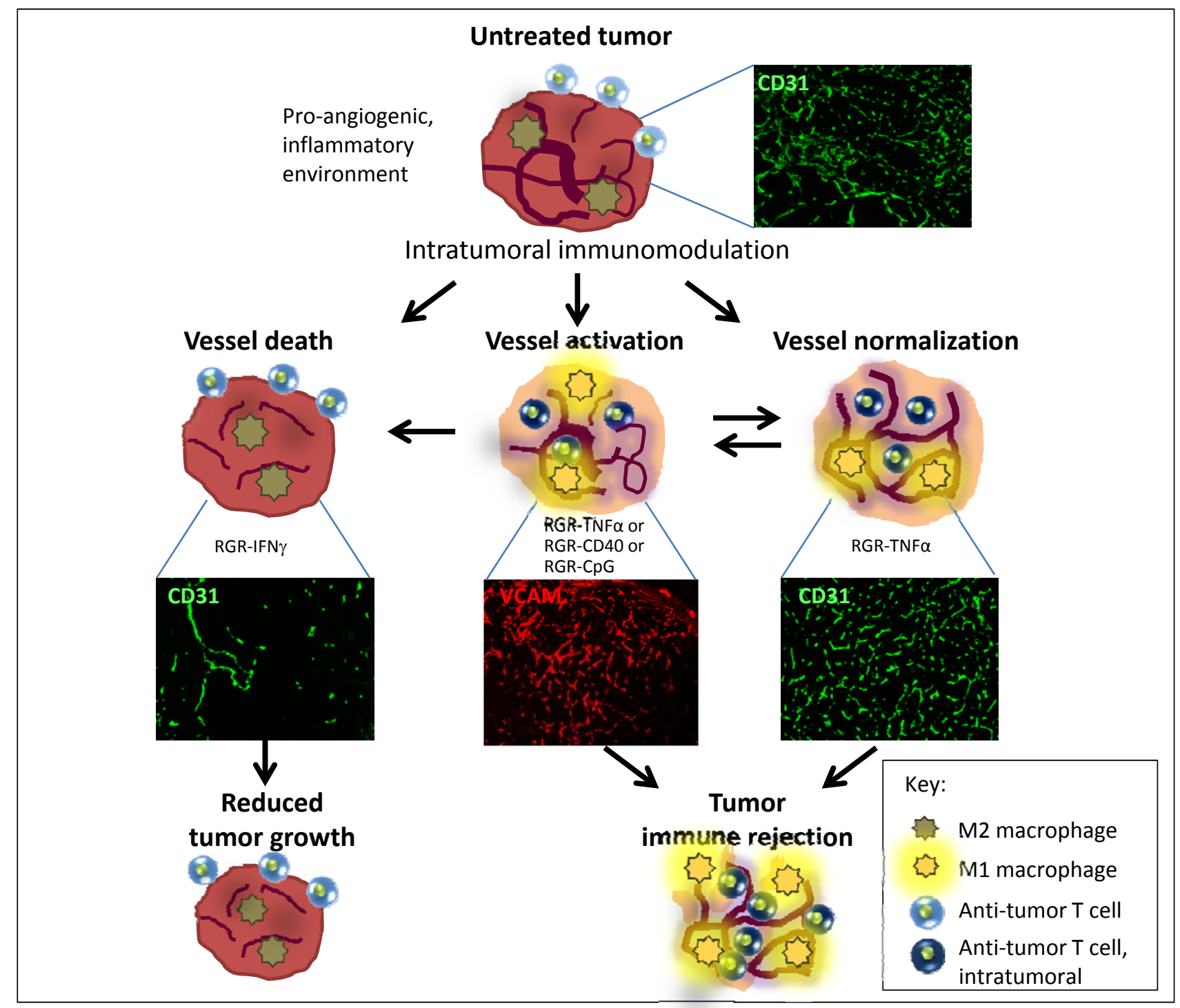




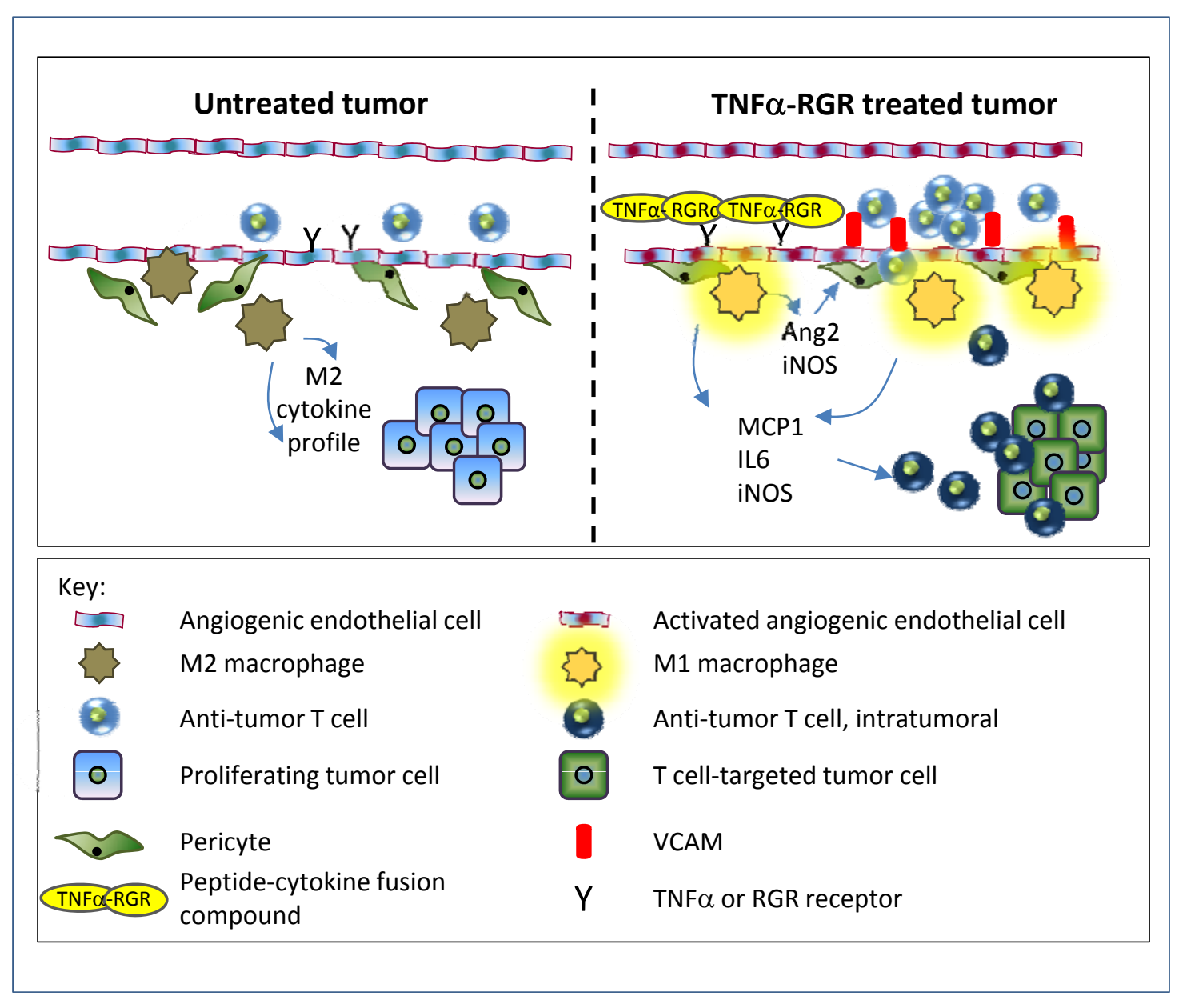


Table 1: Vessel targeting and anti-tumor cytotoxicity ${ }^{\mathrm{a}}$

\begin{tabular}{|c|c|c|c|c|c|c|}
\hline Ligand & Target & Receptor & $\begin{array}{l}\text { Active } \\
\text { compound } \\
\text { (directly } \\
\text { conjugated } \\
\text { to ligand) } \\
\end{array}$ & $\begin{array}{l}\text { Combination } \\
\text { therapy }\end{array}$ & Tumors & Refs \\
\hline \multicolumn{7}{|c|}{ Preclinical models } \\
\hline \multirow[t]{5}{*}{$\begin{array}{l}\text { RGD and } \\
\text { analogues }\end{array}$} & TEC & $\alpha_{v}$ integrins & TNF $\alpha$ & chemotherapy & lymphoma & {$[80]$} \\
\hline & & & IL12 & none & neuroblastoma & {$[81]$} \\
\hline & & & $\begin{array}{l}{ }^{90} \mathrm{Y} \\
(\beta \text {-emitter })\end{array}$ & none & $\begin{array}{l}\text { ovarian cancer } \\
\text { xenograft }\end{array}$ & [82] \\
\hline & & & TRAIL & chemotherapy & $\begin{array}{l}\text { colon cancer } \\
\text { xenografts }\end{array}$ & {$[20]$} \\
\hline & & & $(\text { KLAKLAK })_{2}$ & none & breast cancer xenograft & {$[21]$} \\
\hline \multirow[t]{3}{*}{ NGR } & TEC/TPC & CD13 & TNF $\alpha$ & chemotherapy & $\begin{array}{l}\text { melanoma, lymphoma } \\
\text { xenografts and } \\
\text { transgenic prostate } \\
\text { tumor model }\end{array}$ & $\begin{array}{l}{[27]} \\
{[37]} \\
{[38]} \\
{[83]}\end{array}$ \\
\hline & & & doxorubicin & none & breast cancer xenograft & {$[23]$} \\
\hline & & & tTF & none & $\begin{array}{l}\text { lung carcinoma, } \\
\text { fibrosarcoma, } \\
\text { melanoma } \\
\text { xenotransplants }\end{array}$ & $\begin{array}{l}{[84]} \\
{[85]}\end{array}$ \\
\hline \multirow[t]{3}{*}{ L19 or F8 } & TEC & $\begin{array}{l}\text { ED-B } \\
\text { fibronectin }\end{array}$ & $\mathrm{TNF} \alpha$ & chemotherapy & $\begin{array}{l}\text { fibrosarcoma, colon } \\
\text { carcinoma }\end{array}$ & $\begin{array}{l}{[86]} \\
{[87]}\end{array}$ \\
\hline & & & IL2 & chemotherapy & lymphoma, melanoma & [80] \\
\hline & & & IL12 & chemotherapy & $\begin{array}{l}\text { teratocarcinoma, } \\
\text { lymphoma, colon } \\
\text { carcinoma }\end{array}$ & $\begin{array}{l}{[88]} \\
{[89]}\end{array}$ \\
\hline \multicolumn{7}{|c|}{ Clinical trials } \\
\hline \multirow[t]{3}{*}{ NGR } & TEC/TPC & CD13 & $\mathrm{TNF} \alpha$ & none & $\begin{array}{l}\text { hepatocellular } \\
\text { carcinoma (phase II), } \\
\text { colorectal carcinoma } \\
\text { (phase II), malignant } \\
\text { pleural mesothelioma } \\
\text { (phase II) }\end{array}$ & $\begin{array}{l}{[40]} \\
{[90]} \\
{[39]}\end{array}$ \\
\hline & & & $\mathrm{TNF} \alpha$ & $\begin{array}{l}\text { capecitabine- } \\
\text { oxaliplatin }\end{array}$ & $\begin{array}{l}\text { colorectal carcinoma } \\
\text { (phase II) }\end{array}$ & [91] \\
\hline & & & $\mathrm{TNF} \alpha$ & doxorubicin & $\begin{array}{l}\text { solid tumors (phase } \\
\text { Ib), ovarian carcinoma } \\
\text { (phase II) }\end{array}$ & $\begin{array}{l}{[34-} \\
35]\end{array}$ \\
\hline \multirow[t]{2}{*}{ L19 } & TEC & $\begin{array}{l}\text { ED-B } \\
\text { fibronectin }\end{array}$ & $\mathrm{TNF} \alpha$ & none & $\begin{array}{l}\text { solid tumors (phase } \\
\mathrm{I} / \mathrm{II} \text { ) }\end{array}$ & {$[92]$} \\
\hline & & & IL2 & dacarbazine & $\begin{array}{l}\text { metastatic melanoma } \\
\text { (phase II) }\end{array}$ & [93] \\
\hline
\end{tabular}

${ }^{a}$ Abbreviations: CD13, metalloprotease aminopeptidase N; ED-B, extra domain and B splice variant; F8, antibody fragment directed against a specific splice variant of fibronectin; IL2, interleukin 2; IL12, interleukin 12; (KLAKLAK) 2 , apoptosis-inducing peptide; L19, antibody fragment directed against a specific splice variant of fibronectin; NGR, peptide containing the NGR motif (Asn-Gly-Arg); RGD, peptide containing the RGD motif (Arg-Gly-Asp); TEC, tumor endothelial cells; TNF $\alpha$, tumor necrosis factor $\alpha$; TPC, tumor pericytes; TRAIL, TNFrelated apoptosis-inducing ligand; tTF, truncated tissue factor. 
Table 2: Vessel targeting and active immunotherapy ${ }^{\mathrm{a}}$

\begin{tabular}{|c|c|c|c|c|c|c|}
\hline Ligand & Target & Receptor & $\begin{array}{l}\text { Active } \\
\text { compound } \\
\text { (directly } \\
\text { conjugated } \\
\text { to ligand) }\end{array}$ & $\begin{array}{l}\text { Combination } \\
\text { therapy }\end{array}$ & Tumors & Refs \\
\hline \multirow[t]{3}{*}{ RGR } & TEC/TPC & N/A & $\begin{array}{l}\text { CD40 } \\
\text { agonist } \\
\text { antibody } \\
\text { and IL2 }\end{array}$ & $\begin{array}{l}\text { adoptive } \mathrm{T} \text { cell } \\
\text { transfer }\end{array}$ & $\begin{array}{l}\text { pancreatic } \\
\text { neuroendocrine cancer }\end{array}$ & [43] \\
\hline & & & $\begin{array}{l}\text { CpG-ODN } \\
\text { containing } \\
\text { liposomes }\end{array}$ & $\begin{array}{l}\text { adoptive } \mathrm{T} \text { cell } \\
\text { transfer or anti- } \\
\text { tumor } \\
\text { vaccination }\end{array}$ & $\begin{array}{l}\text { pancreatic } \\
\text { neuroendocrine cancer }\end{array}$ & [44] \\
\hline & & & $\mathrm{TNF} \alpha$ & $\begin{array}{l}\text { adoptive T cell } \\
\text { transfer or anti- } \\
\text { tumor } \\
\text { vaccination }\end{array}$ & $\begin{array}{l}\text { pancreatic } \\
\text { neuroendocrine cancer }\end{array}$ & [45] \\
\hline NGR & TEC/TPC & CD13 & $\mathrm{TNF} \alpha$ & $\begin{array}{l}\text { anti-tumor } \\
\text { vaccination or } \\
\text { anti-tumor } \\
\text { vaccination/che } \\
\text { motherapy }\end{array}$ & melanoma & [54] \\
\hline L19 & TEC & $\begin{array}{l}\text { anti-ED-B } \\
\text { fibronectin }\end{array}$ & IL2 & anti-CTLA-4 & $\begin{array}{l}\text { teratocarcinoma, colon } \\
\text { carcinoma }\end{array}$ & [98] \\
\hline
\end{tabular}

Abbreviations: CD13, metalloprotease aminopeptidase N; CD40, TNF receptor superfamily member 5; CpG-ODN, oligodeoxynucleotides with CpG motifs; CTLA4, cytotoxic Tlymphocyte antigen 4; ED-B, extra domain and B splice variant; IL2, interleukin 2; L19, antibody fragment directed against a specific splice variant of fibronectin; NGR, peptide containing the NGR motif (Asn-Gly-Arg); RGR, peptide containing the RGR motif (ArgGly- Arg); TEC, tumor endothelial cells; TNF $\alpha$, tumor necrosis factor $\alpha$; TPC, tumor pericytes; 